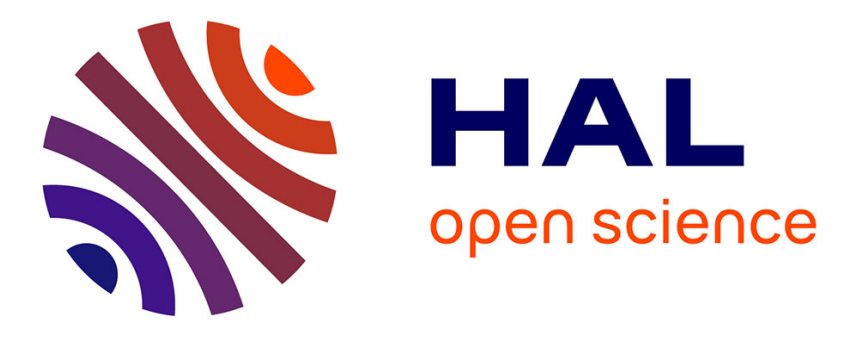

\title{
Helium desorption in 3He implanted tungsten at low energy
}

Pierre-Emmanuel Lhuillier, Aurélien Debelle, Taieb Belhabib, Anne-Lise

Thomann, Pierre Desgardin, Thierry Sauvage, Marie-France Barthe, Pascal Brault, Yves Tessier

\section{To cite this version:}

Pierre-Emmanuel Lhuillier, Aurélien Debelle, Taieb Belhabib, Anne-Lise Thomann, Pierre Desgardin, et al.. Helium desorption in 3He implanted tungsten at low energy. Journal of Nuclear Materials, 2011, pp.504-507. 10.1016/j.jnucmat.2010.12.174 . hal-00688706

\section{HAL Id: hal-00688706 https://hal.science/hal-00688706}

Submitted on 18 Apr 2012

HAL is a multi-disciplinary open access archive for the deposit and dissemination of scientific research documents, whether they are published or not. The documents may come from teaching and research institutions in France or abroad, or from public or private research centers.
L'archive ouverte pluridisciplinaire HAL, est destinée au dépôt et à la diffusion de documents scientifiques de niveau recherche, publiés ou non, émanant des établissements d'enseignement et de recherche français ou étrangers, des laboratoires publics ou privés. 


\section{Helium desorption in 3He implanted tungsten at low energy}

Pierre-Emile Lhuillier ${ }^{1}$, Aurélien Debelle ${ }^{1,2}$, Taieb. Belhabib ${ }^{3}$, Anne-Lise Thomann ${ }^{3}$, Pierre Desgardin ${ }^{1,2}$, Thierry

Sauvage 1,2, Marie-France Barthe 1,2, P. Brault 3 , Yves Tessier $^{3}$

${ }_{1}^{1}$ CNRS, UPR3079 CEMHTI, 1D avenue de la Recherche Scientifique, 45071 Orléans cedex2, France

${ }^{2}$ Université d'Orléans, Faculté des Sciences, Avenue du Parc Floral, BP 6749, 45067 Orléans cedex 2, France ${ }^{3}$ GREMI Polytech'Orléans, 14 rue D’Issoudun, BP 6744, 45067 Orléans Cedex 2, France

Abstract

The behavior of helium in ${ }^{3} \mathrm{He}$ implanted tungsten has been studied using Nuclear Reaction Analysis as a function of the post-implantation annealing temperature. Two different implantation conditions have been investigated: medium energy $(60 \mathrm{keV})$, and low energy $(0.3 \mathrm{keV})$, which exhibit drastically different helium release behavior. In the case of medium energy implantation, desorption starts from $1550 \mathrm{~K}$ and seems to be due to the dissociation of single helium-vacancy complexes $\left(\mathrm{He}-V_{1}\right)$. At $1873 \mathrm{~K}$ the released fraction reaches $75 \%$ that suggests the presence of a second type of helium trapping site. In the case of low energy implantation, desorption is observed from $400 \mathrm{~K}$ (slightly above room temperature) and indicates the presence of shallow helium traps the nature of which is discussed. The released fraction of helium saturates at $\sim 60 \%$ at the temperature of $1473 \mathrm{~K}$ which could be due to helium trapping at single He$V_{1}$ complexes.

\section{Introduction}

Tungsten has been selected as a potential Plasma Facing Material (PFM) in the future nuclear fusion reactors. Its low sputtering yield and its high melting temperature make tungsten the best candidate to withstand the extremely severe conditions encountered in fusion reactors. PFM suffer strong bombardment of the plasma particles such as helium, with energies in the range from few tens of $\mathrm{eV}$ to several keV. Moreover, the neutron irradiation produced by the fusion reaction induces helium introduction by nuclear transmutation and defects creation in the first-wall material. That is why, in the field of materials for fusion, many studies dealing with the behavior of helium implanted in tungsten over a large range of energy have already been carried out [1-3]. In particular, the behavior of helium implanted in tungsten by mean of plasma process or ion accelerator has been widely investigated by several authors $[4,5]$. Recently a study of the desorption behavior of $500 \mathrm{keV}^{3} \mathrm{He}$ implanted ions in tungsten has shown that no helium desorption occurs after post-implantation annealing up to $1773 \mathrm{~K}$ [6].

The aim of the present study is to explore different implantation conditions of energy and fluence and to examine their consequences on the desorption of helium after post-implantation thermal treatment. Precisely two implantation conditions are investigated. In the

\footnotetext{
* Corresponding author at: CNRS, UPR3079 CEMHTI, 1D avenue de la Recherche Scientifique, 45071 Orléans cedex2, France. Tel.: +332 3825 5729; fax: +3323863 0271 .

E-mail address: pierre-emile.Ihuillier@enrs-orleans.fr (P.E. Lhuillier).
}

present paper, the implantation energies of ${ }^{3} \mathrm{He}$ ions $-0.3 \mathrm{keV}$ and $60 \mathrm{keV}$ - are respectively below and above the required energy to produce displacements of $\mathrm{W}$ atoms. Thus the influence of implantation-induced defects is also discussed.

\section{Experimental}

\subsection{Material}

Samples used in the present work are polycrystalline tungsten cut out from cold-rolled $150 \mu \mathrm{m}$ thick commercial foils with a high purity of $99.95 \mathrm{wt} . \%$. The samples have been submitted to a thermal treatment in order to reduce the large concentration of rolling/polishing-induced defects [6,7]. Annealings were performed at $1873 \mathrm{~K}$ for $1 \mathrm{~h}$ under high vacuum $\left(10^{-7}-10^{-6} \mathrm{mbar}\right)$. A positron annihilation study reveals that such type of thermal treatment leads to a quasi-complete defect recovery since the corresponding positron annihilation characteristics are very close to the tungsten lattice ones.

\section{2. ${ }^{3} \mathrm{He}$ implantation}

The helium implantations are carried out with two different conditions of energy and fluence: $60 \mathrm{keV}{ }^{3} \mathrm{He}$ at the fluence of $2 \times 10^{13} \mathrm{~cm}^{-2}$ and $0.3 \mathrm{keV}{ }^{3} \mathrm{He}$ at the fluence of $6 \times 10^{15} \mathrm{~cm}^{-2}$. For convenience the two cases will be respectively denominated as Medium Energy (ME) and Low Energy (LE) conditions in the text hereafter. 
The Medium Energy implantations were performed with the $400 \mathrm{kV}$ electrostatic accelerator of the IPN-Lyon. During the implantations, the temperature of the sample holder was maintained below $330 \mathrm{~K}$. The maximum transmittable energy from the incident ions to the tungsten lattice atoms is higher than $E_{\mathrm{d}}$, displacement threshold energy for the tungsten: $90 \mathrm{eV}$ [8]. The theoretical helium and damage profiles have been calculated with SRIM [9]. The maximum of helium concentration is located at $\sim 132 \mathrm{~nm}$ and reaches $2 \times 10^{-3}$ at.\%. The average damage level over the whole cascade region is estimated to $2 \times 10^{-4} \mathrm{dpa}$.

The Low Energy implantations were carried out by a low pressure plasma process. The sample was immersed in the periphery of a RF Inductively Coupled ${ }^{3} \mathrm{He}$ plasma $\left(2 \times 10^{-3} \mathrm{mbar}, 50 \mathrm{~W}\right)$. In the ICP plasma, the electron energy is low and we can assume that only mono-charged helium ions are created. A bias voltage of $-300 \mathrm{~V}$ was applied to the sample holder in order to accelerate the ${ }^{3} \mathrm{He}$ ions through the sheath. Taking into account the plasma potential $\left(V_{\mathrm{p}}=20 \mathrm{~V}\right)$, the corresponding ion energy is $320 \mathrm{eV}$. The samples have been immersed in the plasma during 3600s under a mean ion flux of $2.5 \times 10^{14} \mathrm{ion} / \mathrm{cm}^{2} / \mathrm{s}$. The surface homogeneity of the helium introduction has been checked and the lateral fluctuations of the measured helium level were below $10 \%$ over the whole surface of the sample holder. Temperature was checked at the backside of the sample holder which is made of a well heat conducting material. A good thermal contact between the samples and the sample holder was ensured (silver paste) and no significant heating was evidenced. For implantation the sample holder was located at the plasma edge, and the interaction of low energy ${ }^{3} \mathrm{He}^{+}$at the reported dose is not expected to induce heating of the surface. Indeed the temperature of the sample was followed and remained in the range between $292 \mathrm{~K}$ and $303 \mathrm{~K}$. It must be noted that the maximum transmittable energy from a $0.3 \mathrm{keV}^{3} \mathrm{He}$ ion to a tungsten atom is of the order of $19 \mathrm{eV}$ which is below the displacement threshold of tungsten $(90 \mathrm{eV})$. This is why no creation of Frenkel pair by direct collision with incident ion - hence no dpa - is expected with these implantation conditions. The maximum of the theoretical implantation profile is located in the first ten nanometers below the surface.

\subsection{NRA and post-implantation thermal treatment}

The helium content of the samples was measured by Nuclear Reaction Analysis (NRA). The implementation of this technique is based on the ${ }^{3} \mathrm{He}(\mathrm{d}, \alpha){ }^{1} \mathrm{H}$ nuclear reaction. The protons emitted during the nuclear reaction and transmitted through the samples are detected at $0^{\circ}$ from the incident deuteron beam. Details about the technique can be found elsewhere [10]. In the case of in situ desorption curve measurement, the sample was heated and the remaining helium content was measured as a function of temperature. All those experiments were carried out on a dedicated apparatus DIADDHEM at the CEMHTI-Cyclotron, Orleans [11,12]. In order to look at any influence of the analyzing ion beam on helium desorption measurements, the evolution of the proton signal has been followed during $8 \mathrm{~h}$. For the reference sample (as-implanted) of the ME implantation set, the signal remained constant over the entire analysis duration, which underlines that the observed helium release cannot be attributed to the probe beam. The influence of the probe beam has also been checked for the reference sample (not submitted to any post-implantation thermal treatment) of the LE implantation conditions. It appears that the probe beam actually induces a slight release of helium. Nonetheless the desorption induced by the analysis beam does not exceed $15 \%$ during a recording of $4 \mathrm{~h}$. As a consequence, this phenomenon should be taken into account in the interpretation of the desorption behavior in the case of LE implantation. However, as it will be shown later, it cannot explain the whole desorption trend observed on this set of samples.

After implantation the samples of the ME implantation set were annealed during $1 \mathrm{~h}$ under vacuum or under $\mathrm{Ar}-\mathrm{H}_{2}$ atmosphere at different temperatures in the range from $1473 \mathrm{~K}$ to $1873 \mathrm{~K}$. The samples implanted in LE conditions have been heated in situ under vacuum, from $300 \mathrm{~K}$ to $1473 \mathrm{~K}$ with a temperature ramp of $\sim 0.7 \mathrm{~K} \mathrm{~s}^{-1}$.

\section{Results and discussion}

\subsection{Post-implantation helium content}

First of all, the total helium content just after implantation is determined by NRA. Within the ME conditions the effective implanted fluence is $(1.89 \pm 0.3) \times 10^{13} \mathrm{~cm}^{-2}$. This value is nearly equal to the implanted amount of helium and points out that the helium has not diffused to the surface and is most likely trapped during implantation.

In the case of LE implantation a huge discrepancy is observed between the expected implanted amount of helium and the value which is effectively measured after implantation. Indeed, the theoretical expected level of implanted helium as regard of the recorded fluence is of the order of $3.9 \times 10^{17} \mathrm{~cm}^{-2}$, whereas the actual amount of implanted helium measured by NRA is $6 \times 10^{15} \mathrm{~cm}^{-2}$. The backscattering probability calculated by SRIM $(\sim 45 \%)$ and the uncertainty about the fluence measurement $(\sim 10 \%)$ cannot explain the difference. Such a wide gap between the expected and the actual fluence is still not clearly understood. Nevertheless some explanations can be suggested. At first we can note that because of the low concentration of pre-existing defects, a substantial fraction of helium is inserted in interstitial site. Therefore they can migrate and reach the surface of the sample without being trapped. This statement is supported by the very low energy of interstitial migration for helium in tungsten which is below $0.25 \mathrm{eV}$ [13-15]. The ejection and release of inserted helium could also be enhanced by the bombardment of the plasma particles itself. Moreover it must be noted that helium implanted at energy below the required energy to displace tungsten atom still induce the formation of vacancy-traps resulting from the "trap mutation" phenomenon [16]. The stress field generated by a growing He interstitial cluster can be relaxed by ejecting a self-interstitial atom (SIA) [14]. The created $\mathrm{He}_{x}-V_{n}$ complex can continue to grow with increasing He filling. Thus it has been suggested by other authors [17] that the saturation of helium retention in similar implantation conditions $\left(0.5 \mathrm{keV}{ }^{4} \mathrm{He}^{+} 10^{17}-10^{19} \mathrm{~cm}^{-2}\right)$ might be due to the creation of a dense array of helium-vacancy complexes within the $\sim 20 \mathrm{~nm}$ under the surface. The expansion of such $\mathrm{He}_{x}-V_{n}$ complexes would be blocked by the stress field of adjacent complexes, hence the saturation of He retention. The phenomenon of retention saturation is also consistent with the formation of bubbles which could be expected in such samples. Indeed growing bubbles which penetrate the top surface would induce a large release of helium and thus would largely reduce the retention rate. One can also say that the grain boundaries are not suppose to play a role in the desorption of helium since they are assumed to be preferential trapping site for helium [18]. Moreover, the mean grain size after annealing was found to be of the order of $20 \mu \mathrm{m}$, thus the ratio of the grain boundary surface on the bulk grain volume is rather low. Nevertheless, some Scanning Electron Microscopy observations were performed and no obvious evidence of the presence of bubbles penetrating the surface was found. Moreover Transmission Electron Microscopy experiments are currently in progress to get an unambiguous response on the presence of helium bubbles on the near surface. 


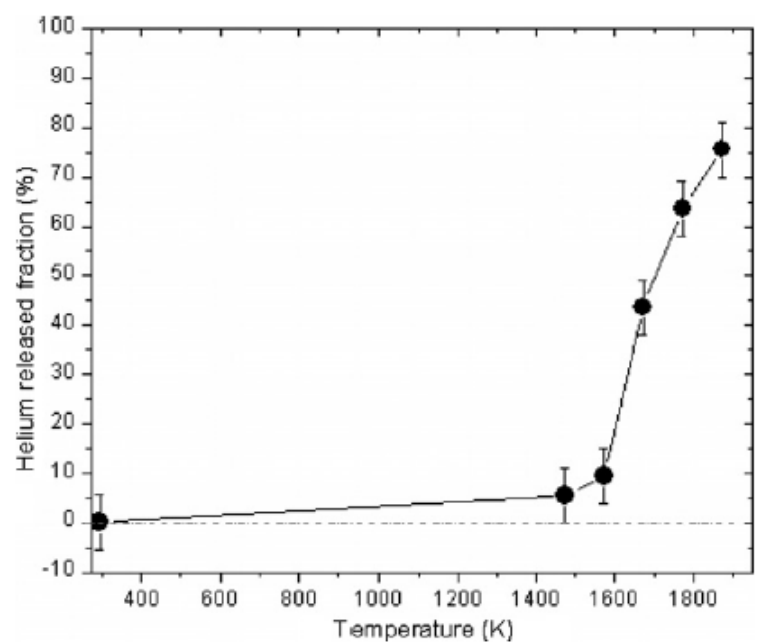

Fig. 1. Helium desorption curve obtained by NRA from $60 \mathrm{keV}{ }^{3} \mathrm{He}$ implanted tungsten at the fluence of $2 \times 10^{13} \mathrm{~cm}^{-2}$ and ex-situ annealing during $1 \mathrm{~h}$ at different temperatures.

\subsection{Helium desorption behavior}

In the case of ME implantations, the helium released fraction in the tungsten samples that have been submitted to ex-situ isothermal annealing up to $1873 \mathrm{~K}$ is presented in Fig. 1. Helium is clearly released when the temperature increases. As shown in Fig. 1, desorption starts from the temperature of $1500-1600 \mathrm{~K}$. Other experimental results [19] and several calculations $[14,20]$ report that this temperature corresponds to the release of helium from monovacancies $\left(V_{1}\right)$. Moreover it has been shown in a previous study that the implantation-induced defects detected by Positron Annihilation Spectroscopy in the track region of $800 \mathrm{keV}^{3} \mathrm{He}$ ions are monovacancies [21]. Furthermore in the latter case the median energy of the primary knock-on atom (PKA) [22] estimated over the whole damage zone is $\sim 500 \mathrm{eV}$. In the present conditions $60 \mathrm{keV}{ }^{3} \mathrm{He}$ ions - the median energy of the PKA is of the order of $300 \mathrm{eV}$ along the ion tracks. Thus it is reasonable to consider that the implantation-induced defects created by $60 \mathrm{keV}{ }^{3} \mathrm{He}$ ions are also monovacancies. In the present work, the estimated concentration of radiation-induced defects is well higher than the concentration of helium (see Section 2.2). Thus we can assume that the probability for a helium atom to meet a monovacancy and so to be trapped is rather high. Therefore the hypothesis of the formation of He- $V_{1}$ complexes during implantation is highly plausible. Those complexes are dissociated at $1500-1600 \mathrm{~K}$ triggering the start of helium release. At $1873 \mathrm{~K}$ the helium released fraction reaches $75 \%$. This result shows that a significant fraction of helium atom is also trapped in other types of defects which act as deeper traps for helium, presumably large $\mathrm{He}_{x}-V_{n}$ complexes.

Indeed, as regard to recent $a b$ initio calculations, the binding energy of a single helium atom with different type of $\mathrm{He}_{x}-V_{n}$ complexes was calculated [23]. It was shown that the binding energy of a single $\mathrm{He}$ atom with a $\mathrm{He}_{x}-V_{n}$ complex increases with the total number of helium atom filling the cluster. In other words the lower the He to vacancy ratio of a $\mathrm{He}_{x}-V_{n}$ complex, the more trapped the He atoms are. In particular above $1873 \mathrm{~K}$, helium might be trapped at $\mathrm{He}_{x}-V_{n}$ complexes with low He to vacancy ratio.

In regard to the LE conditions, the helium desorption behavior is completely different. Fig. 2 represents the released fraction of helium from the sample implanted under the LE conditions as a function of the temperature. The temperature sequence which has been applied to the sample is also displayed in the Fig. 2 (dotted line).

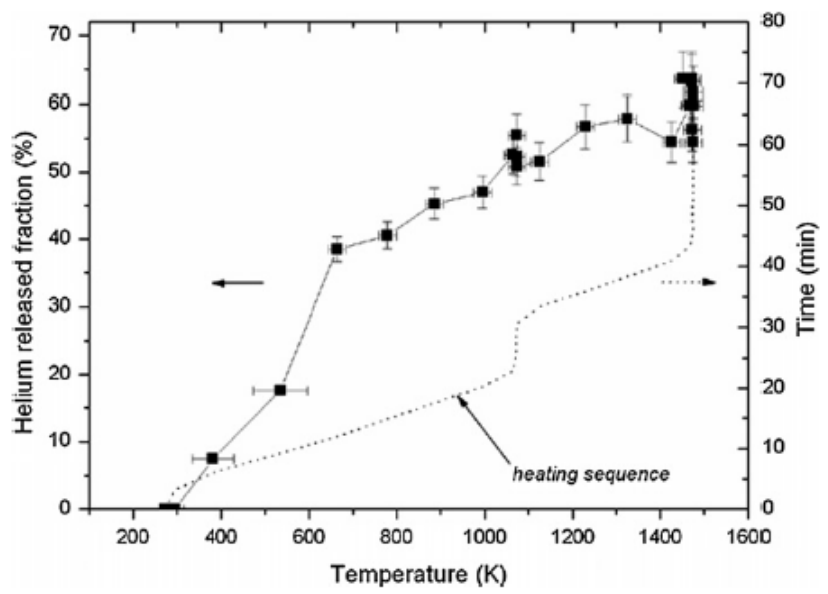

Fig. 2. In-situ Helium desorption curve obtained by NRA from $0.3 \mathrm{keV}{ }^{3} \mathrm{He}$ implanted tungsten at the fluence of $6 \times 10^{15} \mathrm{~cm}^{-2}$ and heated (dotted curve) under the probe beam up to $1473 \mathrm{~K}$.

Desorption is observed from $400 \mathrm{~K}$, slightly above room temperature. The released fraction rapidly increases to $40 \%$ with increasing temperature up to $660 \mathrm{~K}$ in the first $15 \mathrm{~min}$ of the experiments. This important desorption rate is much higher than the contribution of the analysis beam-induced desorption (see Section 3.1). This trend suggests the presence of very shallow trapping sites for helium. Since a moderate increase of temperature above room temperature is sufficient to trigger the release of helium, we can assume that the binding energy of helium with such shallow traps in rather low. This early release step was already reported by Lee et al. [24] by THDS measurements with similar helium introduction conditions $\left(500 \mathrm{eV} \mathrm{He}+10^{17}-10^{20} \mathrm{~cm}^{-2}\right)$. Different shallow trapping sites were proposed to explain this release step. It could presumably correspond to helium release from interstitial loops [16] or desorption of helium trapped in the tungsten deformed lattice in the stress field generated in the vicinity of $\mathrm{He}_{x}-V_{n}$ [25]. From $700 \mathrm{~K}$ to $1473 \mathrm{~K}$ the release fraction of helium slightly increases with the temperature. The maximum released fraction reaches $\sim 60 \%$ at $1473 \mathrm{~K}$. This helium fraction which remains trapped up to $1473 \mathrm{~K}$ was not identified by previous THDS studies [17,24]. This is due to the method of determination of the total He retention by THDS and to the fact that authors did not apply temper atures above $1400 \mathrm{~K}$. It suggests that a substantial part of helium ( $40 \%)$ is located in deeper trapping sites such as helium-vacancy complexes $\left(\mathrm{He}_{x}-V_{n}\right)$ which are not supposed to be dissociated at $1473 \mathrm{~K}$ as discussed above. The influence of probe beam-induced desorption in the slight increase of the desorption from $600 \mathrm{~K}$ to $1473 \mathrm{~K}$ cannot totally be disregarded.

\section{Summary}

The helium desorption behavior in tungsten is observed by Nuclear Reaction Analysis within two different implantation conditions: $60 \mathrm{keV}^{3} \mathrm{He}$ at the fluence of $2 \times 10^{13} \mathrm{~cm}^{-2}$ and $0.3 \mathrm{keV}^{3} \mathrm{He}$ at the fluence of $6 \times 10^{15} \mathrm{~cm}^{-2}$. The two desorption curves exhibit drastically different trends as a function of the implantation energy and fluence. In the case of $60 \mathrm{keV}{ }^{3} \mathrm{He}$ implantation, desorption starts from $1500-1600 \mathrm{~K}$. This desorption threshold corresponds to the release of He from tungsten monovacancies. Furthermore up to $1873 \mathrm{~K}$ the desorbed fraction reaches $75 \%$, suggesting the trapping of helium atoms at deeper traps which can presumably be $\mathrm{He}_{x}-V_{n}$ complexes with a high helium to vacancy ratio.

In the case of $0.3 \mathrm{keV}{ }^{3} \mathrm{He}$ implantation, the primary knock-on atom energy is far below the displacement threshold for Frenkel pair formation. The helium desorption is observed at very low temperature just above room temperature. The desorption step is 
due to the shallow trapping of helium at various trapping sites which could be interstitial loops or trapping sites of the deformed tungsten lattice. Nevertheless a substantial fraction of helium remains strongly trapped since the released fraction of helium reaches $60 \%$ at $1473 \mathrm{~K}$. At this temperature, helium can be trapped at larger $\mathrm{He}_{x}-V_{n}$ complexes.

\section{Acknowledgement}

Authors would like to thanks Ch. Viaud (CEA - Cadarache) and Ch. Peaucelle (INP-Lyon) for the thermal treatments and the implantations.

This work was carried out within the framework the European Fusion Development Agreement and the French Research Federation for Fusion Studies. It is supported by the European Communities under the contract of Association between Euratom and CEA. The views and opinions expressed herein do not necessarily reflect those of the European Commission. The present work was also financially supported by the Energy Program of CNRS, France.

\section{References}

[1] V.N. Chernikov, A.P. Zakharov, J. Nucl. Mater. 165 (1989) 89-100. [2] P. Nambissan, P. Sen, Solid State Commun. 71 (1989) 1165-1167.
[3] D. Nishijima et al., J. Nucl. Mater. 313-316 (2003) 97-101.

[4] N. Yoshida et al. J. Nucl. Mater. 337-339 (2005) 946-950.

[5] T. Hino, Y. Yamauchi, Y. Hirohata, J. Nud. Mater. 266-269 (1999) 538541.

[6] A. Debelle, M.F. Barthe, T. Sauvage, R. Belamhawal, A. Chelgoum, P. Desgardin, H. Labrim, J. Nucl. Mater. 362 (2007) 181.

[7] A. Debelle, M.F. Barthe, T. Sauvage, J. Nucl. Mater. 376 (2008) 216 221.

[8] Standard Practice for Neutron Radiation Damage Simulation by ChargeParticle Irradiation, Annual Book of SATM Standards, Vol. 12.02, Philadelphia, 1996.

[9] J. Ziegler, J. Biersack, U. Littmark, The stopping and range of ions in solids, Pergamon, New York, 1985.

[10] F. Pászti, Nucl. Instrum. Methods Phys. Res., Sect. B 66 (1992) 83-106.

[11] G. Martin, T. Sauvage, P. Desgardin, P. Garcia, G. Carlot, M.F. Barthe, Nucl. Inst. Methods Phys. Res., Sect. B 258 (2007) 71-478

[12] T. Sauvage et al., J. Nucl. Mater. 327 (2004) 159-164

[13] J. Amano, D.N. Seidman, J. Appl. Phys. 56 (1984) 983-992.

[14] W.D. Wilson, C.L. Bisson, M.I. Baskes, Phys. Rev. B 24 (1981) 5616.

[15] CS. Becquart, C. Domain, Phys. Rev. Lett. 97 (2006) 196402.

[16] H. Iwakiri et al., J. Nud, Mater. 283-287 (2000) 134-1138.

[17] H.T. Lee et al., J. Nucl. Mater. 363-365 (2007) 898-903.

[18] H. Zhou et al., Nucl. Inst. Methods Phys. Res. B 267 (2009) 3189-3192.

[19] S.T. Picraux, F.L. Vook, J. Nucl. Mater. 53 (1974) 246.

[20] E.V. Kornelsen, A.A. Van Gorkum, J. Nucl. Mater. 92 (1980) 9-88.

[21] P.E. Lhuillier, M.F. Barthe, P. Desgardin, W. Egger, P. Sperr, Phys. Status Solidi, accepted manuscript.

[22] R.S. Averback, J. Nucl. Mater. 216 (1994) 49-62.

[23] C.S. Becquart, C. Domain, J. Nucl. Mater. 385 (2009) 223-227.

[24] H. Lee et al., J. Nucl. Mater. 360 (2007) 196-207.

[25] M. Tokitani et al., J. Nucl. Mater. 363 (2007) 443-447. 